\title{
Revanches de la bêtise dans L'Idiot de la famille
}

\author{
Jean-François Louette
}

\section{OpenEdition}

Journals

Édition électronique

URL : http://journals.openedition.org/recherchestravaux/219

DOI : 10.4000/recherchestravaux.219

ISSN : 1969-6434

\section{Éditeur}

UGA Éditions/Université Grenoble Alpes

\section{Édition imprimée}

Date de publication : 15 octobre 2007

Pagination : $29-48$

ISBN : 978-2-84310-111-3

ISSN : 0151-1874

\section{Référence électronique}

Jean-François Louette, "Revanches de la bêtise dans L'Idiot de la famille ", Recherches \& Travaux [En ligne], 71 | 2007, mis en ligne le 15 avril 2009, consulté le 08 septembre 2020. URL : http://

journals.openedition.org/recherchestravaux/219; DOI : https://doi.org/10.4000/recherchestravaux 219 
Jean-François LouetTE

Université de la Sorbonne - Paris IV

\title{
Revanches de la bêtise dans L'Idiot de la famille
}

\author{
Ô Bêtise! Bêtise! Bêtise humaine \\ aux innombrables faces, aux innombrables \\ métamorphoses, aux innombrables apparences! \\ Maupassant, «Jour de fête»
}

Stupidity : tel est le titre, alléchant, d'un ouvrage récent ${ }^{1}$. Y sont commentés des textes de Nicolas de Cuse (De docta ignorantia), Rousseau, Kant, Jean Paul (Von der Dummbeit), Marx, Nietzsche, Hölderlin, Valéry (la bêtise n'était pas son fort), Musil (Über die Dummbeit, le dernier de ses essais publiés), Barthes. Flaubert y trouve naturellement sa place. Mais l'auteur oublie radicalement et l'idiot selon Georges Bataille, et le dialogue de Sartre avec ledit Flaubert.

Pourtant les dates parlent d'elles-mêmes. En I 980 Sartre meurt en laissant inachevé L'Idiot de la famille. Cent ans plus tôt, Flaubert mourait en laissant inachevé Bouvard et Pécuchet. Ce roman, Sartre disait le trouver « assommant ${ }^{2}$ ». Sans aller jusqu'à prêter aux deux « cloportes » des mœurs particulières, on peut néanmoins soupçonner, dans la mesure où Sartre voit, comme de juste, dans ce roman l'histoire de " deux autodidactes» (IDF, t. II, p. 2003), que le couple formé par Bouvard et Pécuchet a pour fils l'Autodidacte de La Nausée. Ce dernier s'instruit par ordre alphabétique : pour reprendre un mot de Thibaudet, dans son Gustave Flaubert de 1935, qui parlait des deux « vieillards abécédaires » que sont Bouvard et Pécuchet, l'Autodidacte est lui aussi un homme abécédaire, lui aussi un «écolier hors de saison ». Tels pères, tel fils.

I. Avital Ronell, Stupidity, 200 I, trad. fr., Stock, 2006.

2. «Sartre parle de Flaubert», Michel Sicard, Essais sur Sartre, Galilée, I 989, p. I6 5. 
Inutile de revenir sur l'intérêt constant de Sartre pour Flaubert : on sait, par exemple, que le nom «Bouville» est un hommage au mot «Bovary», etc. En revanche, l'attention que Sartre porte à la sottise, à l'idiotie, à la bêtise, est moins connue, quoique elle aussi fort ancienne. Lorsque, dans L'Invitée, Beauvoir peint Pierre Labrousse (personnage composé à partir de Dullin et de Sartre), qui fait l'idiot au café - «ses yeux étaient exorbités et il mâchait réellement le tabac » à même sa pipe, pour divertir Xavière ${ }^{3}$-, on devine les pitreries auxquelles Sartre pouvait se livrer. Dans son œuvre on trouve de quoi composer une intéressante galerie d'idiots : outre l'Autodidacte, et certains des « acolytes » décrits dans les Carnets de la drôle de guerre, il y a, dans un état plus grave, l'idiot du début des Mouches, ou encore, plus bucolique, le berger provençal et analphabète du Sursis, Gros-Louis, qui descend à Marseille et, quand on lui lit son livret militaire, s'y découvre mobilisable ${ }^{4}$. Sur un plan plus théorique, c'est dans les Cabiers pour une morale, écrits en 1947-1948, que se trouve une analyse de la sottise et de la bêtise, entre lesquelles Sartre consciemment refuse de distinguer's. On oppose parfois la bêtise, où il y aurait un réseau serré de significations toutes faites, et l'idiotie, qui serait composée de vide, de vent, de silence ${ }^{6}$. Au rebours Sartre fait valoir le paradoxe de la sottise/bêtise, qui est d'être un «vide pesant ${ }^{7} »$.

La présente étude, j'avais un temps pensé l'intituler « La famille d'idiot dans L'Idiot de la famille ». Ce génitif spéculatif, outre que sa bêtise introduisait, par consonance, à mon objet, permettait de jouer sur un double sens. D'une part, un tel titre évoquait la thèse centrale de Sartre, selon laquelle c'est sa famille qui rend Gustave idiot (Sartre manifestement en écrivant L'Idiot ne cesse de se féliciter d'avoir eu une mère aimante, et pas de père). D'autre part, il annonçait une interrogation sur la famille du mot, et du concept «idiot». Projet que je conserve : je vais tenter de rassembler divers termes apparentés, idiotie (dès le titre), bêtise (objet de $n$ pages bien délimitées), mais aussi hébétude, autisme, ou retard (au sens où l'on parle d'un enfant retardé). Il s'agit de marquer comment le livre répond continûment à son titre. Aussi tenterai-je d'en suivre le mouvement, en le simplifiant, et en mettant l'accent sur un ultime renversement, qui advient dans le tome III. La notion de retard, Sartre va en effet la détourner vers l'Histoire, en lui donnant un nom grec (bystérésis), et s'évertuer à montrer

3. Gallimard, I 943 , p. $250-25$ I.

4. Sartre se moque un peu de Giono, ou de l'idiot Gagou dans Colline, quoiqu'il peigne Gros-Louis de manière empathique, et qu'il veuille avec lui poser une question d'importance : de quel droit impliquer le berger des Hautes-Alpes dans une guerre qui semble ne le concerner en rien?

5. Gallimard, I 983 , p. $314-338$.

6. Valérie Deshoulières, Métamorphoses de l'idiot, Klincksieck, 2005, p. 84-89.

7. Cabiers pour une morale, éd. citée, p. 3 I9. 
que par son idiotie Flaubert est en avance sur son époque, dont il forme un génial raccourci. Du coup on quitte le champ de la famille... Et il devenait nécessaire de choisir un autre titre : je décrirai, dans L'Idiot, les différentes revanches de la bêtise.

\section{Idio(t)-syncrasie de Flaubert}

Quelle est l'attitude de Sartre, face à l'idiotie ? Ni médicale, ni philosophique, mais empathique, c'est-à-dire fidèle à la fascination que Flaubert éprouva pour elle.

\section{En suivant Flaubert}

Dans le champ de la médecine, c'est Esquirol, un élève du célèbre médecin aliéniste Pinel, qui introduisit le terme d'idiotie comme concept nosographique, dans son Traité des maladies mentales, en I $838^{8}:$ l'idiot n'est pas l'imbécile, puisque chez ce dernier, il demeure un peu de sensibilité, de mémoire, et d'intelligence ; chez l'idiot, l'instinct domine toutes les facultés, la parole n'existe même pas. Pour ce qui est de la philosophie, on se souvient que Nietzsche dans Le Gai Savoir (I 882) intitule ainsi le fragment $328:$ « Faire du tort à la bêtise ». Voilà depuis Socrate une des tâches de la philosophie... et le mot sera repris par Gilles Deleuze.

Or ce sont là deux positions d'extériorité un peu trop commodes. Contre le discours médical, Sartre ne différencie que dans une faible mesure l'idiotie et la bêtise. Il tient que l'idiot parle, et même écrit. Il l'a fait parler dans Le Sursis, en donnant la parole à Gros-Louis. Il le fera écrire en la personne de Gustave. Contre le propos de Nietzsche, il sait bien que nous portons tous notre poids de bêtise. Il prend la peine de la définir : « cette masse ténébreuse d'affolement, d'incompréhension terrorisée, de peur, d'obstination, de mauvaise foi et d'ignorance qui, sous le nom de bêtise, est pour chacun l'indice de son aliénation » (IDF, t. I, p. 830). Il risquera même, huit cents pages plus loin, ce truisme puissant : « tout homme est bête à ses heures de la même façon que tout homme est mortel ».

Non, ce qui inspire Sartre, c'est le rapport de Flaubert lui-même à la bêtise. Les textes de Gustave ne manquent pas qui l'éclairent. À vingt ans, il est en pleine crise de bêtise, si l'on en croit deux lettres de i 84I que cite Sartre9 : «Quant à moi, je deviens colossal, monumental, je suis bœuf, sphinx, butor,

8. Voir Jackie Pigeaud, Aux portes de la psychiatrie, Aubier, 200 I, p. 339 et suiv. 9. IDF, t. II, p. I66r. 
éléphant, baleine, tout ce qu'il y a de plus énorme, de plus empâté et de plus lourd, au moral comme au physique. Si j'avais des souliers avec des cordons, je serais incapable de les nouer. Je ne fais que souffler, hanner (sic), suer et baver» (7 juillet I 84I). Et deux mois et demi plus tard : «Je m'ennuie... m’ennuie... m'ennuie... je suis bête, sot, inerte... je n'ai pas la vigueur nécessaire pour remplir trois feuilles de papier. Depuis un mois que je suis à Trouville, je ne fais absolument rien que manger, boire et dormir et fumer » (2 I septembre I 84I). Une telle immersion dans la bêtise suscite une réaction chez Flaubert, celle qu'il prêtera à Bouvard et Pécuchet : «Alors une faculté pitoyable se développa dans leur esprit, celle de voir la bêtise et de ne plus la tolérer » (ch. VIII). Leur roman sera donc une « encyclopédie de la bêtise humaine » (Flaubert dans une lettre en I 879), une odyssée de la bêtise... à commencer par celle des deux protagonistes.

La tradition critique, connue de Sartre, insistait déjà beaucoup sur cette fascination/répulsion pour la bêtise. Je me borne à trois points de repère. Paul Bourget notait en I 882 que Flaubert à la fois « subissait » et « recherchait la bêtise » (Essais de psychologie contemporaine), au point de s'en dévorer les pattes sans s'en apercevoir, comme le Catoblépas de La Tentation de saint Antoine: c'est un homme «meurtri par la bêtise ambiante», et en proie à «la rancune de cette bêtise ", au double sens du génitif (la bêtise lui en veut obstinément, et il s'obstine à lui en vouloir). En 1935 Thibaudet, dans son Gustave Flaubert, note dès le premier chapitre que " [l]'expérience de Flaubert consiste à étendre à tous les jours la bêtise du jour de l'an, et à tirer de l'or de ce fumier, à créer de la littérature avec de la bêtise et contre elle, à chercher en elle une excitation et hors d'elle un alibi ». (On retrouvera le jour de l'an dans L'Idiot.) Et dans le chapitre consacré à Bouvard et Pécuchet il propose cette puissante comparaison : «Flaubert savourait, humait, dégustait la bêtise, comme un amateur normand se délecte à un fromage avancé ». Thibaudet n'ose pas tout à fait l'écrire, mais il est clair que pour lui la bêtise est le camembert de Flaubert (on notera la richesse de la rime...). Enfin, dans son article intitulé «La maladie de Flaubert », publié en mars I954 dans Les Temps modernes, article qui fut pour Sartre une incitation à écrire L'Idiot, J.-B. Pontalis avance que « [1] a relation de Flaubert à la bêtise est très exactement la transposition de sa relation à la bourgeoisie »: Sartre retiendra la nécessité d'une approche politique de la bêtise, et c'est là que la nuance se marquera qui distingue bêtise (plus sociale) et idiotie (privée, conformément à l'étymologie).

\section{Renversement}

C'est sur le fond de cette tradition critique qu'il convient de mesurer le renversement introduit par Sartre. Il consiste dans l'hypothèse selon laquelle 
l'obsessionnel de la bêtise était aussi... l'idiot de la famille ! Et le deuxième trait explique le premier : idiot donc obsédé.

En effet, qui a conduit Flaubert à cette fascination pour la bêtise ? Ceux-là mêmes qui de lui ont fait un idiot : sa mère, son père, accessoirement son frère. Ils opèrent une castration double, à la fois sexuelle et intellectuelle. Sexuelle : Sartre, comme il apparaît dans le troisième passage sur Flaubert de Questions de méthode, avait d'abord eu le projet d'étudier la féminité secrète de Flaubert, voué par son enfance à la passivité, et de se demander, posant la question en termes kantiens : «À quelles conditions la féminisation de l'expérience est-elle possible ? ». Intellectuelle, avec le mot lancé par le père, qui marque l'enfant à jamais : tu as du mal à apprendre des lettres, tu es l'idiot de la famille. Donc Sartre, dirais-je, se demande aussi : «À quelles conditions la bêtification de l'expérience est-elle possible ?»-son devenir-bêtise.

Cette double castration s'effectue en trois temps: «la castration originelle s'est deux fois répétée : il y a eu la Chute puis la vocation contrariée » (IDF, t. I, p. 90 I). C'est la mère qui est responsable de ladite castration originelle. Caroline n'apparaissait quasiment pas dans Questions de méthode, où Sartre mettait l'accent sur la «fixation au père» de Gustave; mais dans L'Idiot de la famille, la constitution passive de Gustave est de son fait. Pour Caroline, froide, " presque anaffective » à son égard, il est une "bête étrangère ${ }^{10}$ ». Une bête! Les mères indifférentes (Caroline) font les enfants idiots (Gustave). Les mères aimantes - Anne-Marie - font les enfants géniaux : ainsi Poulou. De là les hébétudes de Flaubert enfant, noyé dans la contingence, incapable de cette activité : apprendre ses lettres. On remarquera que c'est la mère qui détermine le rapport de l'enfant au langage : on est loin pour l'instant de l'ordre symbolique du docteur Lacan. Et Sartre de dépeindre les conséquences que ce difficile apprentissage a sur le rapport de Flaubert au langage : une aptitude à saisir les lieux communs, une fascination pour les calembours (qui manifestent une autonomie du verbe), le sentiment de l'étrangeté de la langue (ainsi dans une expression toute simple comme « Il fait beau »), le goût de rêver sur les mots d'une langue morte, le latin ou le grec, pour autant qu'il ne les comprend pas pleinement, et peut s'adonner à la « rumination hébétée d'un vers virgilien ${ }^{1 \mathrm{I}}$ ».

Ce point de départ - la difficulté à apprendre ses lettres - a été souvent critiqué, jugé fragile ou même inconsistant par les flaubertiens, comme d'ailleurs, il faut bien le dire, l'essentiel de ce qui a trait aux relations entre les membres de la famille Flaubert. Je songe notamment à un article sévère de

ı. Entretien avec M. Contat et M. Rybalka, Situations X, Gallimard, I976, p. 97 ; IDF, t. I, p. I32.

I I. Voir IDF, t. I, p. 620-62 I ; t. II, p. I986-1990 ; t. II, p. 205 5-2060. 
Jean Bruneau, «Jean-Paul Sartre biographe de Flaubert ${ }^{12}$ ». (L'amusant est que cet article n'est lui-même pas exempt d'erreurs factuelles... Ainsi J. Bruneau remarque "l'absence de toute référence au Gustave Flanbert de Thibaudet », où il assure ne pas retrouver l'expression de «m'ont-fait-tort » que Sartre attribue au critique : elle figure pourtant dans la Conclusion du livre de 1935 .) De son côté Josette Pacaly a montré «à quel point il [Sartre] a fait de la famille Flaubert sa propre famille », non sans condamner Sartre pour s'être ainsi perdu dans son propre imaginaire, au point d'en venir, dit-elle, «à la limite de la psychose $\mathrm{e}^{\mathrm{i} 3} »$.

Il me parait plus intéressant de remarquer que dans cette « fable » (pour reprendre le mot de Sartre) de l'analphétisme persistant du jeune Gustave, Sartre transpose au cas de Flaubert un épisode du chapitre $\mathrm{x}$ de... Bowvard et Pécuchet. Les deux « cloportes » ont recueilli les enfants d'un forçat, prénommés Victor et Victorine. Or « Victor distinguait ses lettres, mais, n’arrivait pas à former les syllabes. Il en bredouillait, s'arrêtait tout à coup, et avait l'air idiot ». Sic! Je n'invente rien! Ainsi le jeune Gustave rejoint, par la médiation de Sartre, un personnage du dernier roman de Flaubert. Victor, c'était lui. Un doute me saisit : et si l'hypothèse qui fonde le début de L'Idiot ne venait pas de témoignages fragiles (comme on l'a souvent reproché à Sartre), mais si elle était bien plutôt tirée de cet ultime roman ? Une fois de plus, Sartre lirait les personnages de Flaubert comme autant de figures de leur auteur.

La première répétition de la castration subie par Gustave advient, on l'a dit, lorsque son père, qui ne se reconnaît qu'en Grand Frère Achille, prononce le mot vertigineux : tu es l'idiot de la famille ; c'est la «malédiction paternelle », «fait idiosyncrasique et purement familial », la première Chute de Flaubert $^{14}$. La deuxième répétition se produira lorsque Achille-Cléophas contrariera la vocation d'acteur de son fils, où il ne verra que pur cabotinage. Flaubert dans les deux cas se défend en se faisant un enfant imaginaire. Il se laisse aller à ses rêveries, à ses hébétudes. Il s'imagine en artiste. Cette passivité rêveuse, il arrive à Sartre de la nommer le cogito nihiliste de Flaubert ${ }^{15}$. Un tel cogito implique entre autres que Gustave prenne, par orgueil de

I 2. Dans Lectures de Sartre, Claude Burgelin éd., Presses universitaires de Lyon, I 986.

I 3. Sartre au miroir, Klincksieck, I980, p. 32 I et p. 443.

I4. Voir IDF, t. I, p. 380 et t. III, p. 38-39.

I 5 . Voir IDF, t. III, p. 406 : « on me pense donc on m'existe; je ne pense pas donc je n'existe pas ; je sais que je ne suis pas ce que je suis persuadé d'être et que je ne vois pas ce que je prétends voir, donc je suis. Je suis comme le fou qui sait qu'il est fou et ne peut s'empêcher de l'être ; je suis le triomphe de la conscience sur les fantasmes et l'impuissance de celle-là à dissiper ceux-ci; je suis le rêveur qui ne cesse de penser : je suis le rêve d'un rêve et qui, pour autant, ne parvient pas à se réveiller». 
rebond, c'est-à-dire réaction à l'humiliation primitive, une option cosmique et déréalisante : considérer toutes choses d'un point de vue global, celui d'une intuition généralisante, selon laquelle, puisque mon propre père me condamne, c'est que le monde tout entier est la proie du Mal. Cette option à son tour détermine l'opposition, dans les romans de Flaubert, entre l'intrigue (sujet apparent, par exemple l'affaire des époux Delamare, une empoisonneuse, à l'origine de Mme Bovary), et le sujet réel (le glissement inéluctable vers la mort, l'horreur de vivre). Il faut le souligner : l'analyse de cette option de déréalisation et de mise à mort du réel donne une unité à l'ensemble de l'œuvre, et fournit peut-être «l'axe de lecture le plus cohérent qu'on ait jamais proposé des textes flaubertiens ${ }^{16} »$.

\section{Le " martyr de la Bêtise "}

Trois fois castré, Flaubert est voué à devenir « le martyr de la Bêtise ». Avec sa majuscule de majesté, l'expression apparait au terme de l'analyse que Sartre consacre à la Bêtise. De cette analyse la place, à la fin de la Première partie, « La constitution », fait par elle-même sens. La bêtise en effet s'oppose à la liberté, qui, elle, dominera dans le mouvement de "personnalisation », lequel occupe la Deuxième partie. La bêtise entretient un double rapport, passif et actif, avec l'oppression. Passif: dans les Cahiers pour une morale, Sartre partait d'un mot de Marcel Jouhandeau («le sot n’a pas toujours l'air opprimé qui lui convient»), et il en faisait ressortir la justesse, montrant qu'en principe la sottise vient au sot par les autres comme une oppression ${ }^{17}$. Le sot peut en effet toujours dire : j'ai fait ce que j'ai pu; en revanche, c'est autrui qui dit : mais ce n'était pas assez, tu n'as pas compris ce qu'il fallait faire (et que moi je comprends). La sensibilité toute particulière de Flaubert à la bêtise révèle en ce sens sa sensibilité à l'oppression subie - de fait, c'est la situation douloureuse que lui a faite son père. Mais dans L'Idiot advient un renversement par rapport aux Cabiers pour une morale: Sartre insiste sur la « force positive » de la bêtise selon Flaubert, et du coup «le sot devient un oppresseur» (IDF, t. I, p. 6 6 2) actif. C'est sans doute à ce point qu'intervient l'opposition entre idiotie et bêtise. Sartre ne la thématise jamais véritablement ; mais on comprend bien que l'idiot Flaubert n'est jamais un oppresseur. Son idiotie est privée : sur elle pèse le poids de la bêtise commune, mais la réciproque n'est pas vraie.

Comment agit la Bêtise ? Usant d'une terminologie hégélienne, Sartre en analyse les deux faces : comme substance, et comme négativité.

I6. Claude Burgelin, «De Sartre à Flaubert ou la genèse d'un roman vrai », Revue d'Histoire littéraire de la France, I98 I, 4/5, p. 699.

i7. Op. cit., p. $33 \mathrm{I}$. 
La Bêtise comme substance implique et la cérémonie (le nouvel an comme "bêtise sentimentale ", les processions comme "bêtise matérialiste de dogmes figés »), et le langage (les lieux communs et les idées reçues, « substance impersonnelle anonyme »). Je n'entre pas dans les détails. Je me bornerai à noter qu'aux exemples de Sartre dans le tome I de L'Idiot on pourrait ajouter celui de cette autre cérémonie qu'est la distinction bourgeoise. Dans le tome III de L'Idiot, Sartre nous annonce qu'il va se contenter de rappeler " brièvement la genèse et le sens de cet habitus, l'ayant décrit ailleurs ${ }^{18}$ ". Brièvement? L'analyse s'étend de la page 243 à la page $250 .$. Sartre montre que la distinction est la cérémonie d'une éthique anti-naturaliste, par laquelle le bourgeois sacrifie en lui le besoin pour asseoir sa domination sur l'homme du besoin qu'est l'ouvrier. Ailleurs? À la fois dans la Critique de la raison dialectique $^{19}$, et dans la première partie de l'article « La conscience de classe chez Flaubert ", paru dans Les Temps modernes en mai ig66 (en juin ig66 la deuxième partie s'intitule «Bêtise et bourgeoisie »). De la distinction Sartre ne disait pas dans sa revue tout à fait la même chose que ce qu'on lit dans L'Idiot: il insistait sur la vulgarité de la distinction. Qui veut faire l'ange fait la bête : «la distinction, en jetant l'interdit sur les besoins, les détermine comme vulgarité dans la personne même qui les condamne et, comme on ne peut les masquer sans cesse, ils reparaissent et la vulgarité triomphe; elle atteint la distinction en elle-même en l'obligeant à chercher des compromis avec le corps ». Il y a donc un " tourniquet harassant du vulgaire et du distingué », qu'illustre au mieux des Esseintes, distingué par excellence qui finit par «se nourrir à la sonde et par l'anus ${ }^{20} »$.

Pour ce qui est du langage, trois brèves remarques. Commençons par noter que lieux communs et idées reçues forment un système de paroles qui circule de lui-même et partout : bêtise rime avec hantise, la bêtise ne se tait pas, elle prend la parole et ses aises, elle se répand, brouillard pesant et bavard ${ }^{2}$. D'autre part, Sartre souligne que nous ne cessons de lutter contre la matérialité, l'opacité inerte qui tend à saisir, à pétrifier notre parole : « nous sommes tous bêtes dans la mesure où chaque parole prononcée comprend en elle la contre-finalité qui la dévore ». C'est ainsi (l'exemple est présenté dans Questions de méthode) que le mot de Nature vole à Sade sa pensée, qui est une anti-physis. Notre lutte perpétuelle, « ce combat douteux » contre les lieux

I 8. Je rappelle en passant que l'une des pièces du dialogue de Bourdieu avec Sartre, qui a occupé toute la vie du penseur béarnais, s’intitule La Distinction (Minuit, 1979).

i9. Voir t. I, p. 848-853.

20. Les Temps modernes, mai i 966, p. I942-1944; IDF, t. III, p. 305.

2 I. Voir Clément Rosset, Le Réel. Traité de l'idiotie, Minuit, I 977 , rééd. 2004, p. I 43, sur l'interventionnisme de la bêtise. 
communs, voilà ce qui définit l'intelligence. Enfin, il faut souligner qu'il y a une dimension polémique dans la définition que Sartre donne de la bêtise langagière. Il n'insiste pas sans raison sur le mot de système. La bêtise, c'est "le langage s'organisant en lui [Flaubert] selon des liens propres", et à ce niveau, il «ne croit pas qu'on parle: on est parlé» (IDF, t. I, p. 622). On voit bien que Sartre vise le structuralisme et le lacanisme : pensées non pas du langage, mais de la bêtise du langage.

À l'égard de la bêtise-langage, l'attitude de Flaubert est double. D'un côté, il est tout particulièrement apte à la capter. Car, en raison de sa constitution passive, il saisit d'abord la matérialité du langage. Elle le fascine comme un bloc compact et plein. Il est tentant de se laisser pétrifier par elle, affecter d'inertie, de renoncer à l'inquiétude de la conscience : la bêtise ne vous fera jamais défaut, " elle est, écrit Flaubert dans une lettre, de la nature du granit, dure et résistante », elle soutient qui s'y confie, et relie a minima les hommes entre eux. On songe à la statue d'Impétraz dans La Nausée, qui incarne à la fois cette fascination par la dense inertie de la bêtise, et la gloire comme « minéralisation supérieure ». Céder à la bêtise permettrait de réaliser le vœu exprimé dans La Tentation de saint Antoine : être la matière. Mais d'un autre côté, Flaubert voudrait s'en purifier; il sait bien que sa correspondance fourmille de «lieux communs et prudhommeries », qui ne peuvent que raviver le cuisant souvenir de la malédiction paternelle; il sent aussi que dans l'affrontement avec la bêtise est en jeu une lutte contre son être bourgeois. D'où toute la série des procédés de mise à distance de la bêtise dans ses romans : italiques, style indirect libre, montage alterné d'opinions reçues qui ainsi se déconsidèrent réciproquement, etc.

La deuxième et dernière grande forme de bêtise, la bêtise comme négativité, prend le visage de l'analyse, qui ramènerait les grands sentiments, la poétisation romantique de la réalité, à leurs composantes élémentaires, ordinaires et triviales. Oui, mais ce positivisme scientiste, à jamais incarné dans le chirurgien Achille-Cléophas, et surtout dans le pharmacien Homais, est la «bêtise de l'intelligence »; ou, plus radicalement encore, « la pire bêtise, c'est l'intelligence $^{22} »$. Méditons bien ce triste résultat : l'intelligence n'est pas le moins du monde indemne de la Bêtise, elle peut même en représenter le point ultime. Cette bêtise de l'intelligence comme analyse, c'est la première revanche de la bêtise. Elle advient à l'intérieur du monde de Flaubert, sur le plan de la vision du monde, et de la satire. Du coup Flaubert s'exclut du Savoir par haine de son géniteur, et Sartre lit Bowvard et Pécuchet comme meurtre du père tardivement osé : meurtre du regard analytique, de la Science.

22. Voir IDF, t. III, p. 608 et t. I, p. 33 I. 
Pour qui s'inquiète de l'intelligence, rappelons que Sartre indique ailleurs qu'il n'est pas d'intelligence sans synthèse, entendue comme puissance d'inventer des rapports nouveaux ${ }^{23}$. Mais une telle critique de l'intelligence n'est pas sans exemples. On pourrait la retrouver chez Bataille, avec d'autres attendus, puisqu'il s'agit de rabaisser le savoir au nom du non-savoir, d'où cette formule paradoxale de Méthode de méditation (I 947) : « la bêtise [...] est la fenêtre ». Ou chez Foucault, qui à la fin de "La Bibliothèque fantastique », texte de I964 sur La Tentation de saint Antoine, note que dans Bouvard et Pécuchet jouent un rôle fondamental « le moutonnement des ouvrages dans l'espace gris de la bibliothèque ", "la prolifération indéfinie du papier imprimée ${ }^{24}$ ». L'intelligence s'imprime, elle devient livres. Mais à trop s'imprimer elle ne fait plus impression. Tout cela Sartre l'a déjà vu et suggéré dans La Nausée : on en revient à l'Autodidacte.

\section{Idio(t)-logie de Flaubert}

Flaubert enfant idiot, Flaubert adulte obsédé par la bêtise. Mais de cette bêtise et de cette idiotie, que fait-il, dans le processus de personnalisation qui est le sien ? Pour répondre à cette question il faut préciser le statut de l'idiotie. Elle revêt dans L'Idiot de la famille une triple valeur, somatique, ontologique et poïétique.

Collégien, lycéen, Gustave s'abandonne à des crises d'hébétude. Il est alors au pire de lui-même, comme le manifeste la manière dont il décrit sa maladie psychique de i 84I, dans Novembre. Détruisant en lui-même la pensée, il se laisser aller à ce que Sartre nomme un «devenir-chose du sujet percevant : plus d'Ego, la délivrance »; ce mouvement vers une «mystique de l'abrutissement » se retrouve dans le plaisir pris à jouer le mendiant de Nevers (Sartre parodie Hiroshima mon amour de Duras, publié en I960), un épileptique rencontré au bord de la mer : « il jouit de bafouiller, de bêtifier, de se jeter sur le dos, de se tordre dans les spasmes »; «bavant et délirant », Gustave inquiète son père ${ }^{25}$. Et plus encore lors de la crise de Pont-l'Évêque en i 844 : Flaubert s'y fait gisant pétrifié, matérialité inerte ; en cette Chute il se livre tout entier à un endormissement hystérique. L'Idiot est un grand livre sur l'incubation par l'hébétude, physiologique et rêveuse à la fois.

Incubation de quoi ? Tout ensemble, de l'univers, de l'imaginaire, et de l'écriture. On l'a dit : la bêtise permet de satisfaire le vœu d'être la matière,

23. Voir IDF, t. I, p. 489.

24. Dits et écrits, t. I, Gallimard, «Quarto », 200I, p. 337-338.

25. Voir IDF, t. II, p. I740, 1742 et 1744 . 
formulé à la fin de La Tentation de saint Antoine (et qui s'oppose au début, où l'ermite veut être esprit). "Bêtise: "être la matière", coïncider avec elle au point de ne jamais rêver» (IDF, t. III, p. 3 I 4). La bêtise permet donc de satisfaire un fantasme ontologique de totalité, celui de Flaubert en Tunisie, préparant Salammbô : " redevenir nature et se rejoindre à l'univers grandiose du paganisme » (IDF, t. II, p. 2II3). Elle forme le symétrique exact de la conscience de survol, à laquelle Flaubert recourt souvent, et qui elle aussi vise l'infini (voir la section intitulée "L'absentéisme »). Ce goût de la totalité s'exprime encore dans la figure du Garçon, formation par laquelle Flaubert donne carrière à la fois à son désir d'être un acteur, à sa risibilité, qu'il transforme, non sans masochisme, en comique ${ }^{26}$, et enfin à son désir du tout : aussi le Garçon, qui ne supporte pas qu'il y ait du sens où que ce soit, est-il présenté comme un « grand idiot cosmique » (IDF, t. II, p. I 3 I 3).

La vertu cosmique de la bêtise se paie du risque du silence : la matière ne parle guère. Heureusement elle a aussi une valeur poḯtique. Et par un nouveau renversement Sartre montre que la sensibilité idiote à l'égard du langage fonde la faculté de littérature. Une fois que son père a contrarié sa vocation d'acteur comique, Gustave, renvoyé à ses hébétudes, entre dans une phase d'autisme, défini comme « forme extrême de la passivité» (IDF, t. II, p. I 377 ). Mais cette idiotie commence à se dessiner de manière plus positive, parce que Gustave rêve sur des phrases ou des expressions, comme "Choléra à Calcutta ", et que ces ruminations imaginaires affectent sa perception du langage. En elles l'idiot-syncrasie subie commence à devenir une idiosyncrasie créatrice. Flaubert renverse son « rapport négatif au langage en conception positive du style » (IDF, t. II, p. I998). C'est parce qu'il a eu du mal à apprendre ses lettres qu'il devient sensible à la matérialité du langage, à l'idiosyncrasie des mots : pourquoi y a-t-il deux $l$ et deux $t$ à mille-pattes ? S'arrêter sur cette donnée, c'est pouvoir inventer d'en faire à la fois un poids sur sa propre langue, et un moyen d'exprimer le grouillement de l'insecte, comme Sartre fit dans La Nausée. L'idée nait en Flaubert d'exploiter cette matérialité non-signifiante pour dépasser les significations par un sens indirect : la forme sera l'expression indirecte de l'idée. Le style naît parce que Gustave est apte à ne pas considérer le langage comme un ensemble pratique, et à établir une « dialectique constante du sens et de la signification » (IDF, t. II, p. I994). C'est ainsi que Flaubert retourne son idiotie en génie. Au fond, il s'agit de ceci : « objectiver dans le langage son indisable idiosyncrasie » (IDF, t. II,

26. Risibilité que Sartre commente dans son entretien de 1976 avec Michel Sicard en disant que Flaubert est « ironisable », et que lui rit en le lisant, et que L'Idiot donc doit faire rire par moments, pour rendre le goût du vécu de Flaubert. 
p. 200I). L'idiot-syncrasie deviendra ainsi idio(t)-logie (et non idéologie). Telle est la deuxième revanche de la bêtise, qui se produit dans le style même.

Si le Victor de Bowvard et Pécuchet avait bien du mal à apprendre des lettres, c'est sa sœur qui donne l'exemple de cette sensibilité à la lettre du langage, à sa matérialité : «Victorine posait des questions. D'où vient que $c h$ dans orchestre a le son d'un $q$ et celui d'un $k$ dans archéologie ? On doit par moments joindre deux voyelles, d'autres fois les détacher. Tout cela n'est pas juste. Elle s'indignait ». Si elle n'en restait pas à ce moment négatif, qui est déjà découverte de la riche opacité de la langue, Victorine préfigurerait l'écrivain selon Sartre. Peut-être sera-t-elle tout au moins une bonne lectrice : " la lecture d'une œuvre littéraire, chez Flaubert, n'est jamais agressive, elle ne se réduit pas aux significations abstraites » (IDF, t. II, p. I 7 I 3). Lire agressivement : réduire aux idées, anéantir le «matériau verbal», dépasser « la phrase vers sa signification rigoureuse »; lire littérairement : prendre en compte simultanément les deux niveaux, de la signification directe et du sens indirect.

Une telle conception du style justifierait trois digressions. On a d'abord envie de s'attarder sur ces pages très belles où Sartre s'avoue comme un rêveur de mots, dont il invente avec jouissance l'idiosyncrasie. Ainsi pour le mot Amboise, qui renverrait à ambroisie, framboise, boisé, Blois, etc., ou pour le mot si riche de Constantinople qui doit «nous présentifier» la vieille ville byzantine qu'il désigne. En ce mimologisme indirect parce que rêveur une « fonction imageante » vient singulariser, idiotiser la « fonction signifiante ». On ne devient pas écrivain sans savoir imaginer chaque mot, c'est-à-dire « se fasciner sur lui, et, sans même le voir précisément, le prendre comme tremplin du rêve, saisir sa forme, son goût, sa couleur, sa densité, son visage [...] comme des révélateurs de son être caché, c'est-à-dire de la présence immanente du signifié dans le signifiant ${ }^{27}$ ». Sartre pratique ici un très curieux amalgame théorique : non seulement par "signifié », il entend ce que les linguistes nomment le référent, mais il opère une synthèse qu'on pourrait ainsi résumer : Lanson + Thibaudet + Lévy-Bruhl + Husserl. De Gustave Lanson il retient l'opposition, dans L'Art de la prose (1 908), entre la prose pratique (une vitre qui renvoie à la chose), et la prose d'art (qui, sensible à leurs physionomies, à leurs beautés physiques, traite les mots comme des matières sonores et colorées, comme dans le vers), pour en faire la distinction entre fonction signifiante et fonction imageante des mots. D'Albert Thibaudet il conserve l'accent mis sur la " phrase-image » et les «constructions imitatives » chez Flaubert, bref sur une écriture qui souvent peint ce dont elle parle. À Lucien

27. Voir IDF, t. I, p. 936-940 et t. II, p. 2012. 
Lévy-Bruhl il prend l'analyse de ce qui dans la mentalité primitive remplace le principe de causalité, à savoir un principe de participation mystique (occulte) : tout poète, tout écrivain serait une espèce de primitif perpétué, qui veut que son langage, son style, participent mystérieusement des choses. Enfin, c'est de Husserl que viennent les notions de visée et d'analogon, déjà employées en I 940 dans L'Imaginaire.

Une deuxième digression replacerait cette analyse du mimologisme indirect de Gustave, et de la fonction imageante du style, dans le cadre plus général des réflexions de Sartre sur la littérature. On a souvent noté qu'en évoquant «ce lien quasi sensuel au langage qui fait les vrais écrivains », Sartre, au soir de sa vie, corrige le début fracassant et tranchant de Qu'est-ce que la littérature ?: il montre que le vrai prosateur lui aussi fait de la poésie, c'est-à-dire prend les mots pour des choses. Il renoue donc, par-delà l'intellectuel engagé, avec le petit lecteur qu'il fut. Car dans Les Mots il s'est présenté lui aussi en enfant confronté à la matérialité des lettres, non sans se souvenir du beau chapitre «Alphabet» de Biffures (1948), livre dont Lévinas avait donné un compte rendu dans Les Temps modernes en juin I 949. Enfant incapable, par exemple, de comprendre la fin de Madame Bovary ${ }^{28}$, et donc lui aussi un idiot de la famille à ses heures, à la fois idiot et prodige.

Une troisième digression possible porterait sur les fonctions de l'idiotie en littérature. Je songe au mouvement Dada, à Bataille (lutter contre la prison de la rationalité, surpasser Dada, se révolter contre la société : il y a une esquisse de politique de l'idiotie chez Bataille). Ou à Jacques Rivière, référence moins attendue : pourtant le fameux article de I9I 3 sur "Le roman d'aventures » célèbre la «stupidité » du vrai romancier, qui ne sait pas où il va, qui n’a pas la perspicacité de l'auteur d'un roman d'analyse, qui est débordé par son œuvre, en une "maladresse positive » (et Rivière de convoquer, parmi d'autres exemples, Les Possédés).

Mais revenons. Tout paraît aller pour le mieux : Flaubert fait de son idiotie même le point de départ de son génie. Nouvelle revanche éclatante de la bêtise. Revanche $\mathrm{n}^{\circ} \mathrm{I}:$ il est aussi une bêtise de l'intelligence (analytique). Revanche $\mathrm{n}^{\circ} 2:$ il faut être un idiot face aux lettres de l'alphabet pour devenir un génie des belles-lettres. Mais ce n'est pas tout.

28. C'est aussi le roman qu'il ne pourra pas expliciter, ne publiant pas les notes qui lui auraient été consacrées, et auraient dû former le quatrième volume de L'Idiot. Si bien que son parcours d'écrivain est pris entre cette stupeur initiale et ce silence final, qui fait sens : disant le sens de l'écriture pour lui, Sartre se serait fermé à la littérature... Et il s'y refuse malgré la cécité qui la lui ôte. 


\section{La dernière spirale de la bêtise}

Le cas de Flaubert permet à Sartre de revenir sur une question qui l'a toujours préoccupé : celle des décalages entre temporalité d'un sujet et temporalité de son époque. Il va tenter de les penser grâce à la notion d'bystérésis : issu du grec bustereîn, être en retard, le terme désigne dans le langage de la physique le retard de l'effet sur la cause. Sartre le prend en ce sens scientifique dans L'Étre et le Néant, avec la valeur de rémanence du passé (un clou tordu puis redressé conserve une modification de ses molécules qui explique sa fragilité); puis il va l'historiciser pour l'appliquer à Flaubert, dans Questions de méthode ${ }^{29}$. Contre les marxistes, qui veulent mettre en rapport direct (de détermination ou de reflet) une œuvre et un état de la société, Sartre soutient qu'il faut une médiation, celle de l'enfance de l'écrivain. Une œuvre exprime donc à la fois une situation et des significations contemporaines (elle est synchroniquement homogène à son temps), et un état récent mais dépassé de la société, celui qu'a intériorisé l'écrivain dans sa jeunesse (en fonction de la diachronie de la temporalisation humaine). C'est cette expression de l'antérieur qui est baptisée bystérésis. Ainsi Madame Bovary en I 857 est à la fois le grand roman du Second Empire (ne serait-ce que par sa date de parution), et un roman qui « exprime sous un masque à une génération dégoûtée du romantisme les désespoirs post-romantiques d'un collégien de i 830 »; or ces désespoirs coïncident avec le pessimisme des lecteurs bourgeois de i 857 : en ce sens, Flaubert paraîtra « en avance sur son époque [...] parce qu'il est en retard sur elle $3^{\circ} »$. Il exprime comme par un génie divinatoire ce désespoir présent de i 857 parce que c’est son propre désespoir passé d'apprenti-auteur post-romantique.

Sartre reprendra et affinera ce modèle explicatif dans Les Mots, en I964, avec le thème du handicap de quatre-vingts ans qui fut le sien, et des idées en cours sous Louis-Philippe dont son grand-père le berça. Sous leur influence Sartre rejette toute la mythologie moderne de l'écriture : à la malédiction de l'écrivain (Baudelaire), au paradigme de l'innovation et de la rupture (Rimbaud), à l'auto-télisme de l'œuvre (Mallarmé), il oppose l'idée qu'un écrivain écrit pour (c'est-à-dire souvent contre) ses contemporains, que les exigences du temps présent informent l'œuvre, que l'artiste trouve son matériau dans le fonds de l'époque ${ }^{31}$.

29. Voir L'Être et le Néant, Gallimard, I943, rééd. I 97 I, p. I 53 et I 5 6- I 57 ; Critique de la raison dialectique, Gallimard, «Bibliothèque des idées », I 960, t. I, p. 58 (et aussi p. 504).

30. Questions de méthode, Gallimard, I960, p. 58.

3I. Voir à ce sujet Benoît Denis, «Retards de Sartre», Études sartriennes, n Io, 2005, notamment p. 202-203. 
Dans L'Idiot, Sartre emploie de nouveau cette notion d'bystérésis. Et il la fait fonctionner dans une double perspective : politique et esthétique. Tout se passe selon Sartre comme si Gustave était né non pas en I 82 I, mais en I77I, « cinquante ans plus tôt que ses contemporains » (IDF, t. I, p. 8I), et c'est en raison de cette bystérésis, de ce décalage vers l'antérieur, que la société du Second Empire lui apparaîtra comme un milieu sécurisant. Pourquoi ? Parce qu'elle est féodale, et que c'est sur le mode de la féodalité que Gustave a vécu, dans son enfance, sa relation à son père, chef d'une famille domestique et non pas conjugale, et homme dont il se sentait le vassal. En ce sens, Gustave «s'est fait pour l'Empire à une époque où celui-ci n'était même pas prévisible »; sa névrose « indique et réclame d'avance le régime du pouvoir personnel comme le seul où le jeune névrosé pourra vivre [...] cette inerte Éternité qu'il nomme son aristocratie »; Flaubert sera séduit par le Second Empire dans la mesure où la hiérarchie militaire, donc féodale, lui représente « la relation d'hommage » (IDF, t. I, p. 459 et 490). La Troisième République n'est pas au programme de sa vie : sa mort sociale a lieu quand elle nait.

Sur le plan esthétique, l'bystérésis présente un visage un peu différent. L'accent est alors mis sur la notion de raccourci. La névrose de Flaubert (son idiotie misanthrope, adaptation singulière et maladive) est aussi son avance par rapport à son époque, c'est-à-dire ce par quoi il la résume en raccourci et en accéléré jusqu'à la fin du Second Empire. Je résume l'analyse de Sartre. Dans le saisissant début du tome III de L'Idiot, revenant sur les deux tomes précédents, il souligne que d'autant mieux il a montré la névrose de Flaubert, d'autant plus difficile il s'est rendu la tâche d'expliquer le succès de Mme Bovary en I 857 . En effet, une œuvre névrotique a quelque chose de fermé sur soi, d'incommunicable. Pour que le succès de Flaubert s'explique, il faut que son roman malgré tout exprime en profondeur son époque. Solution : il y a succès de cette œuvre-névrose parce que l'Esprit objectif de l'époque était lui-même névrosési2. Que demande en effet, plus précisément, le public bourgeois en I 857 ? En apparence une littérature de divertissement (Émile Augier, etc.). En profondeur une littérature qui corresponde à sa haine de l'homme, à sa vision noire des relations entre les humains (entre deux individus, la relation dominante est la réciprocité de haine). Pourquoi cette noirceur? Les causes en sont multiples (atomisme social fondé sur la propriété ; peur des ouvriers dont les bourgeois se savent haïs depuis juin I 848, et donc sentiment de mentir en se prétendant la classe universelle, c'est-à-dire à la fois dégoût des massacrés et

32. L'Esprit objectif d'une époque, «c'est à la fois la somme des ouvrages publiés à la date envisagée et la multiplicité des totalisations effectuées par les lecteurs contemporains » (IDF, t. III, p. 56). 
honte d'avoir été massacreurs ; culte de la distinction ; impression d'avoir été floué et vaincu en I 852 par Napoléon III ; idéologie scientiste). Mais peu importe : Flaubert, qui vit dans la haine de soi, produit cette littérature noire qui permet au bourgeois "l'assouvissement imaginaire de sa haine »; si Flaubert a du succès, c'est qu'il fournit exactement ce qu'il attendait au public des « capacités » : « une humanité qu'on peut här parce qu'elle se donne ellemême pour haïssable à travers les personnages qui la représentent » (IDF, t. III, p. 33 1-332). Ainsi l'idiotie névrotique de Flaubert lui permet de faire l'Art-Névrose que réclame le Second Empire : et par là même c'est la raison de son succès d'écrivain.

À ce moment éclate le paradoxe temporel : une névrose qui se forme dans les années i 830 , et qui rend Flaubert misanthrope dès i 835 , exprime le pathos des bourgeois et des classes moyennes dans les années i 850 . Ainsi la névrose de Flaubert apparaît après I 848 , et surtout après i 852 , comme prophétique. Le retardé de la famille est par là même en avance sur son époque. Si Madame Bovary triomphe, c'est parce qu'au bout de dix pages le lecteur bourgeois de i 857 a trouvé ce qu'il attend : le sentiment d'un naufrage sûr mais indéterminé, et qui est à la fois fascinant, mis à distance comme autre (puisque c'est celui du personnage), et dés-historicisé (puisque le Mal est archétypique). Ainsi Flaubert déréalise "par un mensonge universalisant » la faute de la bourgeoisie : montrant l'échec inéluctable de toute vie à travers celle d'Emma, il dé-culpabilise la bourgeoisie qui a massacré en i 848 . Sartre résume son analyse d'une façon qui montre qu'il n'en méconnait pas le caractère acrobatique : «la Chute de 44 reflète aux lecteurs de 57 leur déchéance de 48 ». Cette chute de 44 a été vécue par Flaubert comme à la fois un i 848 et un i 852 : en lui, l'histoire de la société de son temps se constitue "comme processus accéléré », il est un microcosme en avance sur le macrocosme social, sur son époque. Sa vie se présente comme un oracle parce qu'elle est « un raccourci diachronique de l'évolution générale de la sociétés3 ».

Mesurons la chance de Flaubert : il y a un retard structurel en chacun de nous. Mais tous n'ont pas la bonne fortune de rencontrer une époque qui corresponde à ce retard, le demande comme sa clef et son secret. Si bien que pour la plupart, l'bystérésis implique seulement une assez précoce sortie de l'Histoire: un «ce n'est plus mon temps, je ne m'y reconnais plus »- elle avance, je demeure, solitaire, vieillissant et stupide. Tandis que son idiotie, ce retard individuel, et sa solitude misanthrope donnent à Flaubert une avance géniale.

33. Voir, pour les citations de ce paragraphe, IDF, t. III, p. 420, 428, 437. 
Pour conclure je voudrais prendre un peu de champ, dans deux directions, flaubertienne puis sartrienne.

Il est tout d'abord grand temps de faire intervenir Bouvard et Pécuchet contre Sartre : ce serait la quatrième revanche de la bêtise. C'est-à-dire d'imaginer Gustave Flaubert en lecteur de L'Idiot de la famille. Qu'est-ce qui fait ce livre? L'alliance de l'histoire, de la philosophie et de la dialectique. Or les trois sont critiquées dans Bowvard. La liquidation de l'histoire, et de la biographie (celle du Duc d'Angoulême, tentée par les deux compères), Sartre l'avait luimême reprise dans La Nausée ${ }^{34}$. Or il ne cherche guère à contredire cette liquidation dans L'Idiot, et du coup assume un statut épistémologique faible pour son entreprise (" je l'avoue, c'est une fable », etc). Qu'on sente d'autre part «sur les temporaux de Pécuchet la philosophie et l'enthousiasme, joints à l'esprit de ruse » (ch. x), ne plaide évidemment pas, étant donné ce qu'est Pécuchet, et quel que soit le respect dû à la phrénologie, en faveur de la philosophie. Bouvard d'ailleurs la définit ainsi : «On explique ce qu'on entend fort peu, au moyen de mots qu'on n'entend pas du tout!» (ch. IX). La pratique de la philosophie conduit les deux profonds penseurs au bord du suicide... puis dans les consolations de la religion! Revanche de l'abbé Bournisien, devenu le curé de Chavignolles, l'abbé Jeufroy (nom dans lequel on entendra l'avachissement écœurant d'un «je crois»). Ce passage par la religion ne sera certes que provisoire. Mais quand même. On comprend que le «Dictionnaire des idées reçues », qui devait faire partie du second volume du roman («la copie»), sub verbo « Philosophie » note lapidairement: « Ricaner».

Et quant à la dialectique ? N'oublions pas que Pécuchet tente de présenter à Bouvard la dialectique de Hegel : «l'Absolu c'est à la fois le sujet et l'objet, l'unité où viennent se résoudre toutes les différences ", et du coup «les contradictoires sont résolus », «partout un principe qui divise, un principe qui enchaîne » (ch. IX). Tout cela semble les surpasser assez largement. En Bouvard et Pécuchet triomphe Homais. Il n'y en avait qu'un dans Madame Bovary, et c'était déjà beaucoup : cruellement Flaubert le dédouble. À preuve, si l'on veut, ce fait que le connecteur mais est dans Bouvard et Pécuchet celui qui introduit les objections : c'est l'opérateur central de l'égalisation des propositions, de leur anéantissement par mise en équilibre, sans qu'on puisse trancher en faveur de ce qui alors seulement serait une vérité. Par le mais advient l'envers noir de la « dialectique décapitée » que Sartre loue chez Mallarmé ou chez Merleau-Ponty. La fameuse « égalité de tout» (formule des scénarios de

34. Voir «Désillusions biographiques dans La Nausée », dans J.-F. Louette, Silences de Sartre, Toulouse, Presses universitaires du Mirail, 2002. 
Bouvard) loin de passer par la copule, passe par le mais ${ }^{35}$. Chez Flaubert, une objection n'est jamais un tremplin pour le raisonnement, mais toujours un enterrement de la raison. Leurs contradictions dénoncent les sciences, et homo est travaillé (torturé) par le mais... L’homme est un mais perpétuel, ce qui fait de lui un niais sempiternel.

Paul Bourget l'avait bien vu, dans l'essai de psychologie qu'il consacre à Flaubert : le romancier considère la pensée « comme un pouvoir, non plus bienfaisant, mais meurtrier », c'est elle qui « supplicie » l'homme. Mais Sartre a-t-il surpassé cette crise de la pensée et du savoir ? Rappelons-nous la crise de science sur laquelle s'ouvre La Nausée, et l'un des sens possibles de la gravure Melancholia de Dürer (à quoi bon la géométrie, à quoi bon le savoir ?), et encore le ridicule de l'Autodidacte : dérisoirement voué à l'ambition de totalité, lui aussi introduit des objections par mais, et illustre le mot de Paul Bourget encore (toujours dans son article sur Flaubert) : « la culture s'essayant sur un très grand nombre de cerveaux, la quantité des déchets est formidable ». Sartre voit dans Bowvard et Pécuchet un roman où Flaubert entonne "pêle-mêle un savoir indigeste pour le vomir aussitôt sur ses contemporains » (IDF, t. III, p. 600). Mais on peut aussi vomir des styles; ce que fait $L a$ Nausée, réalisant en un sens le projet qu'indique le dernier scénario de Flaubert pour son roman : «Specimen de tous les styles, agricole, médical, théologique, classique, romantique, périphrases ». Comme Roquentin qui rêve de la claire Italie de Stendhal, peut-être Sartre a-t-il envie d'être chassé loin des brumes de la Culture.

Ironie de la vie : la cécité au soir de son existence lui fermera les livres. Adieu aux bibliothèques, et condamnation de L'Idiot à l'inachèvement. Lequel annule tout l'effort qui précède, si l'on en croit les notes pour le quatrième volume, où Sartre se pose cette question qu'il souligne : «Pourquoi écrire les trois premiers volumes si on ne les retrouve pas à chaque page du quatrième ${ }^{36}$ ?»; mais ni celui-ci ni le cinquième n'ont été écrits ${ }^{37}$. Revanche de l'inintelligence forcée ? Au fond, Claude Mouchard, dans son compte rendu de L'Idiot paru dès I97 I, posait la bonne question : « ne voit-on pas éclater ici l'impossibilité profonde du désir encyclopédique, du rêve de réduction anthropomorphique de la dispersion du savoir ${ }^{38} »$ ?

35. Voir par exemple dans l'édition Folio/classique (1979), p. 349 (« Mais il ne peut y avoir plusieurs religions, puisqu'il n'y a qu'un Dieu»), ou p. 398 («les moineaux purgent les potagers [des vers], mais gobent les cerises »).

36. IDF, t. III, p. 783 .

37. Était même prévu un cinquième volume, dit Sartre en 1976 (voir Essais sur Sartre, éd. citée, p. 145).

38. Critique, n² 295, p. 1048-1049. 
On pourrait ajouter : où est la liberté de Gustave, si sa proto-histoire est prégnante à ce point ${ }^{39}$ ? Ce serait farce : L'Idiot de la famille rendrait Sartre idiot au point qu'il renonce à la liberté... Pourquoi ? À force de s'être plongé dans l'Histoire et d'en avoir regardé l'œil médusant. On touche ici à la cinquième (et dernière, rassurons-nous) revanche de la bêtise. Pourquoi Sartre se lancet-il dans l'entreprise démesurée de L'Idiot, pourquoi se place-t-il ainsi en marge de l'Histoire, fuyant son siècle pour le XIX siècle, délaissant l'engagement pour langagement de Flaubert? Les maos de son entourage le lui reprochaient âprement. Mais n'est-ce point qu'il a entrevu la possibilité d'une bêtise de l'Histoire ? Cette bêtise, Sartre l'avait déjà rencontrée avec la deuxième guerre. Elle est très vivace à la fin des années 1950 : après les désillusions de Bucarest et du rapport Khroutchev, où est passé le sens de l'Histoire? Aussi le philosophe ne peut-il que retrouver la grande problématique flaubertienne de la Bêtise. Face à elle l'agent historique n'est pas en position de force : en témoigne le vertige affolé de Frantz von Gerlach dans Les Séquestrés, qui redoute que l'homme n'ait pas progressé d'un pas depuis Néron. Difficile de faire plus que de nommer cette bêtise : c'est l'objet de l'analyse de la contrefinalité dans la Critique de la raison dialectique. La contre-finalité forme un vol de ma praxis, qui l'oblige à réaliser une fin contraire à celle qu'elle se proposait, et en fait " un acte sans auteur ${ }^{40}$ ». Je voulais la paix - j'ai laissé éclater la deuxième guerre mondiale. Je voulais la liberté - j’ai eu Budapest. Je voulais agir - je suis renvoyé à la passivité. J'ai cru être malin - je n'étais qu'idiot. Il faut imaginer Sartre médusé par la Bêtise de l'Histoire.

Au fond, la même association entre passivité et bêtise vaut à la fois pour l'individu Flaubert, et pour l'Histoire. Il y a de la bêtise dans l'Histoire parce qu'en elle une couche constitutive échappe à l'ordre de la praxis. S'il eût su agir Gustave eût su lire, note Sartre (IDF, t. I, p. 363). Si l'homme pouvait agir sans se faire voler son acte, l'Histoire pourrait être, par lui, à la fois lue et écrite. Mais... elle est est bête et méchante (méchante parce que bête).

Il reste que pour Flaubert on peut aussi être bête et bon. C'est la valeur du personnage de Charles Bovary, et Sartre en souligne le mystère : « il est à la fois un imbécile, un raté, et d'un autre côté il est la bonté »; et de trouver curieux que Flaubert " ait choisi un imbécile pour montrer la bonté ${ }^{41}$ ». Profonde énigme, curieuse association - que l'Histoire de son côté ignore.

39. C'est la question de Gilbert Cohen dans « De Roquentin à Flaubert », Revue de métaphysique et de morale, janvier-mars 1976.

40. La formule est dans une note de L'Idiot de la famille, t. I, p. 390. Voir aussi l'analyse des calembours.

4I. Essais sur Sartre, éd. citée, p. I 59-160. 
Au demeurant, nul n'est assez candide pour ignorer que selon Flaubert « la bêtise consiste à vouloir conclure ». Notons cependant encore ceci : parmi les «actes de naissance de l'art moderne » figure sans doute - Dada ou Duchamp ne nous contrediraient pas - «le droit à la bêtise ${ }^{42} »$. Flaubert et Sartre nous l'accordent intelligemment.

42. Jean-Jacques Schuhl, Ingrid Caven, Gallimard, 2000. Réed. «Folio », p. I95. 